\title{
An Extension of the Carathéodory Differentiability to Set- Valued Maps
}

\author{
Pedro Hurtado $\mathbb{D},{ }^{1}$ Alexander Leones $\mathbb{D}^{1},{ }^{1}$ M. Martelo $\mathbb{D}^{2},{ }^{2}$ and J. B. Moreno $\mathbb{D}^{1}$ \\ ${ }^{1}$ Facultad de Ingenierías, Corporación Universitaria Remington, Calle 51, N 51-27 Medellín-Antioquia, Colombia \\ ${ }^{2}$ Program of Mathematics, Universidade Federal Fluminense, Niteroi, Brazil
}

Correspondence should be addressed to J. B. Moreno; john.moreno@uniremington.edu.co

Received 23 February 2021; Accepted 19 May 2021; Published 1 June 2021

Academic Editor: Victor Kovtunenko

Copyright ( 2021 Pedro Hurtado et al. This is an open access article distributed under the Creative Commons Attribution License, which permits unrestricted use, distribution, and reproduction in any medium, provided the original work is properly cited.

This paper uses the generalization of the Hukuhara difference for compact convex set to extend the classical notions of Carathéodory differentiability to multifunctions (set-valued maps). Using the Hukuhara difference and affine multifunctions as a local approximation, we introduce the notion of $\mathrm{CH}$-differentiability for multifunctions. Finally, we tackle the study of the relation among the Fréchet differentiability, Hukuhara differentiability, and $\mathrm{CH}$-differentiability.

\section{Introduction}

Given $X$ and $Y$ nonempty sets, if for each element $x$ belonging to $X$ is assigned, due to a certain law denoted here by $F$ $: X \rightrightarrows Y$, a set $F(x)$ contained in $Y$, we say that $F$ is a multifunction (we also use the term multivalued function or setvalued map). Among the many and various examples and uses of multifunctions, we can find from those simpler provided by elementary algebra and trigonometry to those appearing, for example, in applications of control theory involving optimal control problems and structural properties of control systems such as stability, controllability/reachability ([1-4]), and perturbation theory for ordinary differential equations in the real Euclidean space $\mathbb{R}^{n}([5,6])$; of particular interest is also studying the differentiability of multifunctions applied to perturbed optimization problems as observed in [7], where it is considered the problem "minimize $f(w, x)$ as $x \in A(w)$ " being $f: W \times X \longrightarrow \mathbb{R} \cup\{+\infty\}$ an arbitrary function, $A: W \rightrightarrows X$ a multifunction with $0 \in \operatorname{dom} A$ and $W, X$ Banach spaces. The concept of differentiability for multifunction has been discussed by many authors([8-13]). In [11], it extends the classical notion of Fréchet differentiability to multifunctions, using affine multifunctions and Hausdorff distance.

Other concepts of differentiability can be found in the literature of classical analysis, as the Carathéodory derivative proposed in [14], the strength of Carathéodory's formulation relies on the concept of continuity, and its proof uses strongly the properties of continuous functions. Another advantage about this formulation is that it does not require the difference quotient present in the Fréchet formulation, which is the key to generalize it to a function of several variables. In [15], it explored some of the advantages of Carathéodory's characterization over Fréchet's characterization proposed in [16].

In this work, motivated by the Carathéodory derivative proposed in [14] and generalization of Hukuhara difference proposed $([9,17,18])$, an extension of the classical notion of Carathéodory differentiability to multifunctions defined on finite-dimensional normed spaces is approached. For this purpose, we assume finite-dimensional normed vector spaces $X$ and $Y$ and consider the family of affine multifunctions (see [11] for details of affine multifunctions and the Fréchet differentiability of multifunctions):

$\operatorname{MAFF}_{0}(X, Y)=\{A: X \longrightarrow \mathrm{bccY} \mid A$ is affine and $A(0)=\{0\}\}$,

where bccY is the collection of all bounded closed convex subsets of $Y$, provided with a suitable metric. Then, we associate the differentiability (Carathéodory) of the multifunc- 
tions to the existence of continuous functions

$$
\phi_{1}, \phi_{2}: X \longrightarrow \operatorname{MAFF}_{0}(X, Y) .
$$

This paper is organized as follows. In Section 2, we present some definitions and basic results of generalized Hukuhara difference and affine multifunction. Section 3 presents our extension of Carathéodory differentiability to multifunctions (see Definition 4), named here as $\mathrm{CH}$-differentiability (Carathéodory Hukuhara differentiability), which is based on the set in (1) and the generalized Hukuhara difference, [9]. Also, we show an example of $\mathrm{CH}$-differentiable multifunctions (see Example 4) and study some properties of the $\mathrm{CH}$ differentiability such as uniqueness, continuity, sum, and composition. Finally, in Section 4, we provided a relation involving the Fréchet differentiability, generalized Hukuhara differentiability, and CH-differentiability (Theorems 7, 8, 9, 10).

\section{Preliminaries}

Let $X$ and $Y$ be finite-dimensional normed vector spaces over the set of real numbers $\mathbb{R}$.

A multifunction $F: X \rightrightarrows Y$ from $X$ into $Y$ assigns to each $x \in X$ a (possibly empty) subset $F(x) \subset Y$.

The sets

$$
\begin{gathered}
\operatorname{dom} F=\{x \in X: F(x) \neq \varnothing\}, \\
\operatorname{gr} F=\{(x, y) \in X \times Y: y \in F(x)\},
\end{gathered}
$$

are called the effective domain and the graph of the multifunction $F$, respectively.

By bccY, we denote the collection of all bounded closed convex subsets of $Y$. The collection bccY equipped with the Minkowsky addition:

$$
A+B=\{a+b: a \in A, b \in B\}(A, B \in \mathrm{bccY})
$$

and multiplication by nonnegative real numbers:

$$
\alpha A=\{\alpha a: a \in A\}(A \in \operatorname{bcc} Y, \alpha \geq 0)
$$

is a semilinear space. The function

$$
d_{H}(A, B)=\inf \left\{\alpha \geq 0: A \subset B+\alpha B_{Y}, B \subset A+\alpha B_{Y}\right\}
$$

where $B_{Y}$ is the unit ball in $Y$, is called the Hausdorff distance, and determines a structure of a metric space on bccY

Remark 1. It is well known that the Minkowsky addition is associative and commutative with neutral element $\{0\}$. If $\alpha$ $=-1$, the scalar multiplication gives the opposite $-A=(-1)$ $A=\{-a \mid a \in A\}$ but, in general,

$$
A+(-A) \neq\{0\},
$$

i.e., the opposite of $A$ is not the inverse of $A$ unless $A=\{a\}$ is a singleton. The first implication of this fact is that, in gen- eral, the additive simplification is not valid, that is $(A+C=$ $B+C) \Phi A=B$ or $(A+B)-B \neq A$.

Remark 2. The partial solution for (7) is the Hukuhara difference, this difference has been introduced as a set $C$ for which $A \ominus B=C \Leftrightarrow A=B+C$, and the important property of $\ominus$ is $A \ominus A=\{0\}$ and $(A+B) \ominus B=A, \forall A, B \in$ bccX. The Hukuhara difference is unique, but it does not always exist (a necessary condition for $A \oplus B$ exist is that $A$ contains a translation $\{c\}+B$ of B.)

A generalization of the Hukuhara difference proposed in [17] aims to overcome this situation.

Definition 1 (see [9]). The generalized Hukuhara difference of two sets $A, B \in \mathrm{bccY}$ (gH-difference for short) is defined as follows:

$$
A \ominus_{g} B=C \Leftrightarrow \begin{cases}(a) & A=B+C ; \\ \text { or }(b) & B=A+(-1) C .\end{cases}
$$

Proposition 1 (see [18]). If $C=A \ominus_{g} B$ exists, it is unique and if also $A \odot B$ exists then $A \ominus_{g} B=A \odot B$.

Remark 3 (see [18]). A necessary condition for $A \ominus_{g} B$ to exists is that either A contains a translation of $B$ (as for $A \odot B$ ) or $B$ contains a translation of $A$. In fact, for any given $c \in C$, we get $B+\{c\} \subseteq A$ from (i) or $A+\{-c\} \subseteq B$ from (ii).

Remark 4 (see [18]). If $A \ominus_{g} B$ exists, then $B \ominus_{g} A$ exist and $B$ $\ominus_{g} A=-\left(A \Theta_{g} B\right)$.

Remark 5 (see Proposition 4 in [9]). The generalized Hukuhara difference exists for any two compact intervals.

The generalized Hukuhara difference has the following properties:

Proposition 2 (see [18]). Let $A, B \in b c c Y$ then

(1) If the gH-difference exists, it is unique and it is a generalization of the usual Hukuhara difference, since $A$ $\Theta_{g} B=A \odot B$ whenever $A \odot B$ exists

(2) $A \Theta_{g} A=\{0\}$,

(3) if $A \ominus_{g} B$ exists in the sense ( $a$ ), then $B !{ }_{g} A$ exists in the sense $(b)$ and vice versa

(4) $(A+B) \ominus_{g} B=A$

(5) $\{0\} \ominus_{g}\left(A \Theta_{g} B\right)=(-B) \Theta_{g}(-A)$,

(6) We have $\left(A \ominus_{g} B\right)=\left(B \ominus_{g} A\right)=C$ if and only if $C=\{0\}$ and $A=B$

From now on, in this paper, we will consider the generalized Hukuhara difference, $\Theta_{g}$. 
2.1. Affine Multifunction and Fréchet Differentiability. Let $X$ and $Y$ be finite-dimensional normed vector spaces over the set of real numbers $\mathbb{R}$.

A multifunction $H: X \longrightarrow$ bcc $Y$ is said to be

convex if

$$
\alpha H\left(x_{1}\right)+(1-\alpha) H\left(x_{2}\right) \subset H\left(\alpha x_{1}+(1-\alpha) x_{2}\right),
$$

for all $x_{1}, x_{2} \in \operatorname{dom} H$ and $\alpha \in[0,1]$;

affine if

$$
\alpha H\left(x_{1}\right)+(1-\alpha) H\left(x_{2}\right)=H\left(\alpha x_{1}+(1-\alpha) x_{2}\right),
$$

for all $x_{1}, x_{2} \in \operatorname{dom} H$ and $\alpha \in[0,1]$.

Proposition 3 (see [11]). An affine multifunction $F: X \longrightarrow$ bcc $Y$ is uniformly continuous on dom $F$ in the Hausdorff sense.

Definition 2. A multifunction $F: X \longrightarrow \operatorname{bcc} Y$ is said to be Fréchet differentiable at a point $x_{0} \in \operatorname{int}(\operatorname{dom} F)$, if there exists an affine multifunction $\mathscr{A}\left(x_{0} \mid \cdot\right): X \longrightarrow$ bcc $Y$ such that $0 \in$ int $\left(\operatorname{dom} \mathscr{A}\left(x_{0} \mid \cdot\right)\right)$ and

$$
\lim _{h \rightarrow 0} \frac{d_{H}\left(F\left(x_{0}+h\right), \mathscr{A}\left(x_{0} \mid h\right)\right)}{\|h\|}=0 .
$$

This definition is equivalent to the following: for any $\varepsilon>0$ there exists $\delta>0$ such that for all $h \in \delta B_{X}$, we have

$$
\begin{aligned}
& F\left(x_{0}+h\right) \subset \mathscr{A}\left(x_{0} \mid h\right)+\varepsilon\|h\| B_{Y}, \\
& \mathscr{A}\left(x_{0} \mid h\right) \subset F\left(x_{0}+h\right)+\varepsilon\|h\| B_{Y},
\end{aligned}
$$

where $B_{X}$ and $B_{Y}$ are unit balls in $X$ and $Y$, respectively. So immediately, we have that $\mathscr{A}\left(x_{0} \mid 0\right)=F\left(x_{0}\right)$.

\section{CH-Differentiability and Some Properties}

In this section, we use the Hukuhara difference and the affine multifunction to build the concept of differentiability of a multifunction.

Let $X$ and $Y$ be finite-dimensional normed vector space over real $\mathbb{R}$, define the space:

$\operatorname{MAFF}_{0}(X, Y)=\{A: X \longrightarrow \mathrm{bcc} Y \mid A$ is affine and $A(0)=\{0\}\}$

Example 1. Let $\rho: X \longrightarrow \mathbb{R}$ be a linear function in $X^{*}$, such that $\rho(0)=0$. Choose the function $F: X \rightrightarrows \mathrm{bcc} Y$ defined by $F(x)=\rho(x) B$, for $B \in \operatorname{bcc} Y$. Then, $F \in M A F F_{0}(X, \operatorname{bcc} Y)$.

In effect, $F(\alpha x+(1-\alpha) y)=(\alpha \rho(x)+(1-\alpha) \rho(y)) B=\alpha$ $F(x)+(1-\alpha) F(y)$.

Proposition 4. For $F, G \in M A F F_{0}(X, Y)$,

$$
\tilde{d}(F, G)=\sup _{\|x\| \leq 1}\left\{d_{H}(F(x), G(x))\right\}
$$

is a metric on $\operatorname{MAFF}_{0}(X, Y)$, where $d_{H}$ is the Hausdorff metric.

Proof.

$$
\tilde{d}(F, F)=\sup _{\|x\| \leq 1}\left\{d_{H}(F(x), F(x))\right\}=\sup _{\|x\| \leq 1}\{0\}=0 .
$$

(1) If $\tilde{d}(F, G)=0$, then for all $x \in X$ such that $\|x\| \leq 1$, we have $d_{H}(F(x), G(x))=0$, thus $F(x)=G(x)$ for $x \in X$ such that $\|x\| \leq 1$. On the other hand, since $F$ is affine and $F(0)=\{0\}$, for $\|x\|>1$, we have

$$
\begin{aligned}
F(x) & =\|x\| \cdot \frac{1}{\|x\|} F(x)=\|x\| \cdot\left(\frac{1}{\|x\|} F(x)+\left(1-\frac{1}{\|x\|}\right)\{0\}\right) \\
& =\|x\| \cdot F\left(\frac{1}{\|x\|} \cdot x+\left(1-\frac{1}{\|x\|}\right) \cdot 0\right)=\|x\| \cdot F\left(\frac{x}{\|x\|}\right) \\
& =\|x\| \cdot G\left(\frac{x}{\|x\|}\right)=G(x) .
\end{aligned}
$$

Therefore, $F=G$.

$$
\begin{aligned}
\tilde{d}(F, G) & =\sup _{\|x\| \leq 1}\left\{d_{H}(F(x), G(x))\right\}=\sup _{\|x\| \leq 1}\left\{d_{H}(G(x), F(x))\right\} \\
& =\tilde{d}(G, F) .
\end{aligned}
$$

(2) If $F, G$, and $H$ are multifunctions, then for each $x \in X$ such that $\|x\| \leq 1$, we have

$$
\begin{aligned}
d_{H}(F(x), H(x)) & \leq d_{H}(F(x), G(x))+d_{H}(G(x), H(x)) \\
& \leq \tilde{d}(F, G)+\tilde{d}(G, H)
\end{aligned}
$$

Therefore, $\tilde{d}(F, H) \leq \tilde{d}(F, G)+\tilde{d}(G, H)$.

Definition 3. Let $X, Y$ be finite-dimensional normed vector spaces over the set of real numbers $\mathbb{R}$. A function $\phi: X \longrightarrow$ $\operatorname{MAFF}_{0}(X, \operatorname{bcc} Y)$ is said to be continuous at $x_{0}$, if given $\varepsilon>$ 0 , there exists $\delta>0$ such that, if $\left\|x-x_{0}\right\|<\delta$, then $\tilde{d}(\phi(x), \phi$ $\left.\left(x_{0}\right)\right)<\varepsilon$

Example 2. Let $x_{0}$ be a fixed point and $x$ in $X$. By the HahnBanach theorem (see [19], page 3.), there is a linear function $\rho_{x}: X \longrightarrow \mathbb{R}$ in $X^{*}$, such that

$$
\rho_{x}(0)=0, \rho_{x}\left(x-x_{0}\right)=\left\|x-x_{0}\right\|_{X}^{2} \text {, and }\left\|\rho_{x}\right\|_{X^{*}}=\left\|x-x_{0}\right\|_{X} \text {. }
$$


Let be fixed $B \in \operatorname{bcc} Y$. By Example 1, the functions $F_{x}$ $: X \rightrightarrows$ bcc $Y$ defined by $F_{x}(h)=\rho_{x}(h) B$ are in $\operatorname{MAFF}_{0}(X$, bcc $Y)$. Now, the function

$$
\begin{gathered}
\phi: X \longrightarrow \operatorname{MAFF}_{0}(X, \operatorname{bcc} Y), \\
x \longrightarrow F_{x},
\end{gathered}
$$

is continuous at $x_{0}$.

In effect, $\phi\left(x_{0}\right)=0$ and

$$
\begin{aligned}
\tilde{d}\left(\phi(x), \phi\left(x_{0}\right)\right) & =\sup _{\|h\| \leq 1}\left\{d_{H}\left(F_{x}(h), F_{x_{0}}(h)\right)\right\} \\
& =\sup _{\|h\| \leq 1}\left\{d_{H}\left(\rho_{x}(h) B, 0\right)\right\} \leq\left\|\rho_{x}\right\|_{X^{*}}=\left\|x-x_{0}\right\|_{X} .
\end{aligned}
$$

Theorem 1. If $\phi, \varphi: X \longrightarrow M A F F_{0}(X, Y)$ are continuous at the point $x_{0}$. Then, $\eta(x)=\phi(x) \Theta_{g} \varphi(x)$ is continuous at $x_{0}$.

Proof. Since $\phi$ and $\varphi$ are continuous at $x_{0}$, given $\varepsilon>0$ and $\alpha$ $\epsilon[0,1]$ there are $\delta_{1}>0$ and $\delta_{2}>0$ such that, if $\left\|x-x_{0}\right\|<\delta$ $=\min \left(\delta_{1}, \delta_{2}\right)$ then $\tilde{d}\left(\phi(x), \phi\left(x_{0}\right)\right)<\alpha \varepsilon$ and $\tilde{d}\left(\varphi(x), \varphi\left(x_{0}\right)\right)$ $<(1-\alpha) \varepsilon$. Thus, for $\|h\|<\delta$ and the definition of affine multifunction, we have

$$
\begin{gathered}
\phi\left(x_{0}+h\right) h \subseteq \phi\left(x_{0}\right) h+\alpha \varepsilon\|h\| B_{Y}, \\
\varphi\left(x_{0}+h\right) h \subseteq \varphi\left(x_{0}\right) h+(1-\alpha) \varepsilon\|h\| B_{Y}, \\
\phi\left(x_{0}\right) h \subseteq \phi\left(x_{0}+h\right) h+\alpha \varepsilon\|h\| B_{Y}, \\
\varphi\left(x_{0}\right) h \subseteq \varphi\left(x_{0}+h\right) h+(1-\alpha) \varepsilon\|h\| B_{Y} .
\end{gathered}
$$

Now, if $x \in \eta\left(x_{0}+h\right)(h)$, from Definition 1 , there is $a \in$ $\phi\left(x_{0}+h\right)(h)$ and $b \in \varphi\left(x_{0}+h\right)(h)$, such that $x=a-b$; thus, from (1) and (7), we have

$$
\begin{gathered}
a=a_{1}+\alpha \varepsilon\|h\| a_{2}, \\
b=b_{1}+(1-\alpha) \varepsilon\|h\| b_{2} .
\end{gathered}
$$

where $a_{1} \in \phi\left(x_{0}\right)(h), b_{1} \in \varphi\left(x_{0}\right)(h)$, and $a_{2}, b_{2} \in B_{Y}$. So

$$
\begin{aligned}
& =a-b=a_{1}+\alpha \varepsilon\|h\| a_{2}-b_{1}-(1-\alpha) \varepsilon\|h\| b_{2} \\
& =\left(a_{1}-b_{1}\right)+\varepsilon\|h\|\left(\alpha a_{2}+(1-\alpha)\left(-b_{2}\right)\right)=x_{1}+\varepsilon\|h\| x_{2} .
\end{aligned}
$$

Then, $x_{1}=a_{1}-b_{1} \in \phi\left(x_{0}\right)(h) \Theta_{g} \varphi\left(x_{0}\right)(h)$, this is, $x_{1} \in \eta($ $\left.x_{0}\right)(h)$ and $x_{2}=\alpha a_{2}+(1-\alpha)\left(-b_{2}\right) \in B_{Y}$, since $a_{2},-b_{2} \in B_{Y}$ and $B_{Y}$ is convex. Therefore, $x \in \eta\left(x_{0}\right)(h)+\varepsilon\|h\| B_{Y}$. In consequence, $\eta\left(x_{0}+h\right) \subseteq \eta\left(x_{0}\right)(h)+\varepsilon\|h\| B_{Y}$. Analogously, $\eta\left(x_{0}\right.$ )$\subseteq \eta\left(x_{0}+h\right)(h)+\varepsilon\|h\| B_{Y}$. Thus, $\eta$ is continuous.

Now, motivated by the Carathéodory's Definition in [14], Definition 1, and Proposition 2, we define the following:
Definition 4. A multifunction $F: X \longrightarrow \mathrm{bcc} Y$ is $\mathrm{CH}$ differentiable (Carathéodory and Hukuhara differentiable) at a point $x_{0} \in \operatorname{int}(\operatorname{Dom} F)$, if there are functions $\phi_{1}, \phi_{2}$ $: V_{x_{0}} \longrightarrow \mathrm{MAFF}_{0}(X, \mathrm{bcc} Y)$ continuous at a point $x_{0} \in V_{x_{0}}$, where $V_{x_{0}}$ is a neighborhood of the point $x_{0}$, such that $\phi_{1}$ ( $\left.x_{0}\right)=\phi_{2}\left(x_{0}\right)$ and

$$
\begin{aligned}
& F\left(x_{0}+h\right) \Theta_{g} F\left(x_{0}\right)=\phi_{1}\left(x_{0}+h\right)(h), \\
& F\left(x_{0}\right) \Theta_{g} F\left(x_{0}+h\right)=\phi_{2}\left(x_{0}+h\right)(h),
\end{aligned}
$$

$\forall h \in X$ such that $x_{0}+h \in V_{x_{0}}$.

Remark 6. Given $F: X \rightrightarrows \mathrm{bcc} Y$. It is natural to ask the following question. Are the functions $\phi^{\prime} s$ in Definition 4 unique, the answer is no, even it does not fit for functions (see [15]). Nevertheless, we have the uniqueness of the differential.

Example 3. The constant function $F: X \rightrightarrows$ bcc $Y$ defined by $F(x)=B$, for $B \in \operatorname{bcc} Y$ is $\mathrm{CH}$-differentiable at $X$. In effect, $F$ $\left(x_{0}+h\right) \Theta_{g} F\left(x_{0}\right)=B \ominus_{g} B=\{0\}=\phi\left(x_{0}+h\right)(h)$, where $\phi\left(x_{0}+\right.$ $h)(h)=\{0\} \forall h \in X$.

Example 4. Let $f: \mathbb{R}^{n} \longrightarrow \mathbb{R}$ be a differentiable function at $x_{0} \in \mathbb{R}^{n}$ and $b \in \mathbb{R}^{m}$. The function $F: \mathbb{R}^{n} \rightrightarrows \mathrm{bcc} \mathbb{R}^{m}$ defined by $F(x)=f(x)\{b\}$, is CH-differentiable at $x_{0}$. In effect, $F\left(x_{0}\right.$ $+h) \Theta_{g} F\left(x_{0}\right)=f(x+h)\{b\} \Theta_{g} f\left(x_{0}\right)\{b\}=\left(f\left(x_{0}+h\right)-f\left(x_{0}\right)\right)$ $\{b\}=\psi\left(x_{0}+h\right)(h)\{b\}$ where $\psi$ exists because $f$ is differentiable, thus, Carathéodory differentiable. Now, consider $\phi$ $: V_{x_{0}} \longrightarrow \operatorname{MAFF}_{0}(X, \mathrm{bcc})$ defined by $\phi(x)(z)=\psi(x)(z)\{b\}$ . Then, $F\left(x_{0}+h\right) \Theta_{g} F\left(x_{0}\right)=\phi\left(x_{0}+h\right)(h)$.

Theorem 2. Let $X, Y$ be two Banach spaces and $F: X \rightrightarrows b c c Y$ be a $C H$-differentiable at $x_{0}$. Suppose that, there are functions $\phi$ and $\varphi$ satisfying the $\mathrm{CH}$-differentiability conditions. Then, $\phi\left(x_{0}\right)=\varphi\left(x_{0}\right)$.

Proof. Since $F\left(x_{0}+h\right) \Theta_{g} F\left(x_{0}\right)=\phi\left(x_{0}+h\right)(h)$ and $F\left(x_{0}+h\right)$ $\Theta_{g} F\left(x_{0}\right)=\varphi\left(x_{0}+h\right)(h)$, for all $h \in V_{x_{0}}$. Now define the function

$$
\eta: V_{x_{0}} \longrightarrow \operatorname{MAFF}_{0}(X, \mathrm{bcc} Y)
$$

by $\eta(x)=\phi(x) \Theta_{g} \varphi(x)$. Observe that $\eta\left(x_{0}+h\right)(h)=\{0\}$. Thus

$$
d_{H}\left(\eta\left(x_{0}\right)(h),\{0\}\right)=d_{H}\left(\eta\left(x_{0}\right)(h), \eta\left(x_{0}+h\right)(h)\right) .
$$

Now, since $\phi$ and $\varphi$ are continuous at $x_{0}$. We have $\eta$ is continuous at $x_{0}$. Whence, given $\varepsilon>0$, there is $\delta>0$ such that if $\|h\|<\delta$, then

$$
d_{H}\left(\eta\left(x_{0}\right)\left(\frac{h}{\|h\|}\right), \eta\left(x_{0}+h\right)\left(\frac{h}{\|h\|}\right)\right) \leq \tilde{d}\left(\eta\left(x_{0}\right), \eta\left(x_{0}+h\right)\right)<\varepsilon .
$$


Now, for each $z \in X$ with $\|z\|=1$ there is $N \in \mathbb{N}$, such that for $n>N$, there exists $h_{n} \in X$, with $\left\|h_{n}\right\|<\delta$ and $h_{n} /\left\|h_{n}\right\| \longrightarrow z$ (just take the sequence on the line segment joining 0 to $z$ ). Hence, we have

$$
\lim _{n \longrightarrow+\infty} d_{H}\left(\eta\left(x_{0}\right)\left(\frac{h_{n}}{\|h\|}\right),\{0\}\right)=0 .
$$

Then,

$$
\eta\left(x_{0}\right)\left(\lim _{n \longrightarrow+\infty} \frac{h_{n}}{\|h\|}\right)=\lim _{n \longrightarrow+\infty} \eta\left(x_{0}\right)\left(\frac{h_{n}}{\|h\|}\right)=\{0\} .
$$

Therefore,

$$
\eta\left(x_{0}\right)(z)=\{0\} \text { and } \phi\left(x_{0}\right)=\varphi\left(x_{0}\right) \text {. }
$$

Lemma 1. Let $F, G: X \longrightarrow b c c Y$ be two multifunctions and $x_{0} \in X$, if $\lim _{x \longrightarrow x_{0}} F(x)=F\left(x_{0}\right)$ and $\lim _{x \longrightarrow x_{0}} G(x)=G\left(x_{0}\right)$. Then

$$
\lim _{x \longrightarrow x_{0}} F(x)+G(x)=F\left(x_{0}\right)+G\left(x_{0}\right) .
$$

Proof. In effect, if $\lim _{x \longrightarrow x_{0}} F(x)=F\left(x_{0}\right)$ and $\lim _{x \longrightarrow x_{0}} G(x)=G\left(x_{0}\right)$, then given $\varepsilon>0$ and $\alpha \in[0,1]$, there exists $\delta>0$, such that if $d\left(x, x_{0}\right)<\delta$. Then, $d\left(F(x), F\left(x_{0}\right)\right)<\alpha \varepsilon$ and $d\left(G(x), G\left(x_{0}\right)\right)$ $<(1-\alpha) \varepsilon$. Hence, we obtain that

$$
\begin{gathered}
F(x) \subseteq F\left(x_{0}\right)+\alpha \varepsilon B_{Y}, \\
G(x) \subseteq G\left(x_{0}\right)+(1-\alpha) \varepsilon B_{Y} .
\end{gathered}
$$

It follows that if $z \in F(x)+G(x)$ then $z=a+b$, with $a \in$ $F(x)$ and $b \in G(x)$, thus, $a=a_{1}+\alpha \varepsilon a_{2}$ and $b=b_{1}+(1-\alpha) \varepsilon$ $b_{2}$, with $a_{1} \in F\left(x_{0}\right), b_{1} \in G\left(x_{0}\right)$, and $a_{2}, b_{2} \in B_{Y}$, so $z=a_{1}+$ $b_{2}+\varepsilon\left(\alpha a_{2}+(1-\alpha) b_{2}\right)$. As $\left(\alpha a_{2}+(1-\alpha) b_{2}\right) \in B_{Y}$, we have $z$ $\epsilon F\left(x_{0}\right)+G\left(x_{0}\right)+\varepsilon B_{Y}$. Thus, $F(x)+G(x) \subseteq F\left(x_{0}\right)+G\left(x_{0}\right)$ $+\varepsilon B_{Y}$. Using $F\left(x_{0}\right) \subseteq F(x)+\alpha \varepsilon B_{Y}$ and $G\left(x_{0}\right) \subseteq G(x)+(1-$ a) $\varepsilon B_{Y}$, similarly, $F\left(x_{0}\right)+G\left(x_{0}\right) \subseteq F(x)+G(x)+\varepsilon B_{Y}$. Therefore

$$
d_{H}\left(F(x)+G(x), F\left(x_{0}\right)+G\left(x_{0}\right)\right)<\varepsilon .
$$

Theorem 3. If $F: X \longrightarrow b c c Y$ is a $C H$-differentiable multifunction at $x_{0} \in X$. Then, $F$ is Hausdorff continuous at $x_{0}$.
Proof. Since $F\left(x_{0}+h\right) \Theta_{g} F\left(x_{0}\right)=\phi\left(x_{0}+h\right)(h)$, we have, from the above proposition,

$$
\begin{aligned}
\lim _{h \longrightarrow 0} F\left(x_{0}+h\right) & =\lim _{h \longrightarrow 0} F\left(x_{0}\right)+\phi\left(x_{0}+h\right)(h)=F\left(x_{0}\right)+\phi\left(x_{0}\right)(0) \\
& =F\left(x_{0}\right) .
\end{aligned}
$$

Theorem 4. Let $F, G: X \longrightarrow b c c Y$ be two $C H$-differentiable multifunctions at $x_{0}$. Then, $\alpha F(x)+\beta G(x)$, with $\alpha, \beta \in \mathbb{R}$, is $\mathrm{CH}$-differentiable at $x_{0}$.

Proof. We have, $F\left(x_{0}+h\right) \Theta_{g} F\left(x_{0}\right)=\phi\left(x_{0}+h\right)(h)$ and $G\left(x_{0}\right.$ $+h) \Theta G\left(x_{0}\right)=\varphi\left(x_{0}+h\right)(h)$, thus

$$
\begin{aligned}
\alpha F\left(x_{0}+h\right)+\beta G\left(x_{0}+h\right) & =\alpha\left(F\left(x_{0}\right)+\phi\left(x_{0}+h\right)(h)\right)+\beta\left(G\left(x_{0}\right)\right. \\
& \left.=+\varphi\left(x_{0}+h\right)(h)\right) \\
& =\alpha F\left(x_{0}\right)+\beta G\left(x_{0}\right)+(\alpha \phi+\beta \varphi)\left(x_{0}+h\right)(h) .
\end{aligned}
$$

Hence, since $(\alpha \phi+\beta \varphi)$ is continuous, the result follows.

Theorem 5. If $: X \longrightarrow X$ is a differentiable function at $x_{0}$ and $F: X \longrightarrow b c c Y$ is a CH-differentiable multifunction at $f\left(x_{0}\right)$, then $F \circ f: X \longrightarrow b c c Y$, is $C H$-differentiable at $x_{0}$.

Proof. Observe that, $F\left(f\left(x_{0}\right)+h\right) \Theta_{g} F\left(f\left(x_{0}\right)\right)=\phi\left(f\left(x_{0}\right)+h\right)$

$(h)$ and $f\left(x_{0}+h\right)-f\left(x_{0}\right)=\psi\left(x_{0}+h\right)(h)$, thus

$$
\begin{aligned}
F \circ f\left(x_{0}+h\right) \Theta_{g} F \circ f\left(x_{0}\right) & =F\left(f\left(x_{0}+h\right)\right) \Theta_{g} F\left(f\left(x_{0}\right)\right) \\
& =F\left(f\left(x_{0}\right)+\psi\left(x_{0}+h\right)(h)\right) \Theta_{g} F\left(f\left(x_{0}\right)\right) \\
& =\phi\left(f\left(x_{0}\right)+\psi\left(x_{0}+h\right)(h)\right)\left(\psi\left(x_{0}+h\right)(h)\right) \\
& =\phi\left(f\left(x_{0}\right)+\psi\left(x_{0}+h\right)(h)\right) \circ \psi\left(x_{0}+h\right)(h) .
\end{aligned}
$$

Theorem 6. If $F \in M A F F_{0}(X, b c c Y)$, then $F$ is a $\mathrm{CH}$ differentiable multifunction at $x_{0}$.

Proof. Let $F \in \mathrm{MAFF}_{0}(X, \mathrm{bcc} Y)$ and $\alpha \in(0,1)$. Choose $h=($ $1-\alpha / \alpha) w$ small, thus

$$
\begin{aligned}
F\left(x_{0}+h\right) & =F\left(x_{0}+\frac{1-\alpha}{\alpha} w\right)=F\left(\frac{1}{\alpha}\left(\alpha x_{0}+(1-\alpha) w\right)\right) \\
& =\frac{1}{\alpha}\left(\alpha F\left(x_{0}\right)+(1-\alpha) F(w)\right)=F\left(x_{0}\right)+\frac{1-\alpha}{\alpha} F(w) \\
& =F\left(x_{0}\right)+F\left(\frac{1-\alpha}{\alpha} w\right)=F\left(x_{0}\right)+F(h) .
\end{aligned}
$$


$(h)=F$.

Now, choose $\phi: V_{x_{0}} \longrightarrow M_{A F F_{0}}(X, \operatorname{bcc} Y)$, defined by $\phi$

\section{CH-Differentiability vs. Fréchet Differentiability and Generalized Hukuhara Differentiability}

The following result is useful for studying the relationship between $\mathrm{CH}$-differentiability and Fréchet differentiability.

Lemma 4. Let $A$ be in $b c c Y$ and $\phi \in M A F F_{0}(X, b c c Y)$. Consider $\varphi: X \longrightarrow b c c Y$ defined by

$$
\varphi(x)=A+\phi(x) .
$$

Then, $\varphi$ is an affine multifunction.

Proof. Let $x_{1}, x_{2} \in X$ and $\alpha \in[0,1]$, then

$$
\phi\left(\alpha x_{1}+(1-\alpha) x_{2}\right)=\alpha \phi\left(x_{1}\right)+(1-\alpha) \phi\left(x_{2}\right)
$$

with $\phi(0)=\{0\}$. On the other hand, since $A=\alpha A+(1-\alpha) A$. We have

$$
\begin{aligned}
\varphi\left(\alpha x_{1}(1-\alpha) x_{2}\right) & =A+\phi\left(\alpha x_{1}+(1-\alpha) x_{2}\right) \\
& =\alpha A+(1-\alpha) A+\alpha \phi\left(x_{1}\right)+(1-\alpha) \phi\left(x_{2}\right) \\
& =\alpha\left(A+\phi\left(x_{1}\right)\right)+(1-\alpha)\left(A+\phi\left(x_{2}\right)\right) \\
& =\alpha \varphi\left(x_{1}\right)+(1-\alpha) \varphi\left(x_{2}\right) .
\end{aligned}
$$

Theorem 7. If $F: X \longrightarrow b c c Y$ is a CH-differentiable multifunction at $x_{0}$, then $F$ is Fréchet differentiable at $x_{0}$.

Proof. Using the CH-differentiability of $F$ at $x_{0}$, there exist $\phi_{1}, \phi_{2}: V_{x_{0}} \longrightarrow \operatorname{MAFF}_{0}(X, \mathrm{bcc} Y)$ continuous functions at $x_{0} \in V_{x_{0}}$, where $V_{x_{0}}$ is a neighborhood at $x_{0}$, such that $\phi_{1}$ ( $\left.x_{0}\right)=\phi_{2}\left(x_{0}\right)$ and

$$
\begin{aligned}
& F\left(x_{0}+h\right) \Theta_{g} F\left(x_{0}\right)=\phi_{1}\left(x_{0}+h\right)(h), \\
& F\left(x_{0}\right) \Theta_{g} F\left(x_{0}+h\right)=\phi_{2}\left(x_{0}+h\right)(h),
\end{aligned}
$$

$\forall h \in X$ such that $x_{0}+h \in V_{x_{0}}$. On the other hand, since $\phi_{1}\left(x_{0}\right)$ is an affine multifunction, using Lemma 4.1,

$$
A(h)=F\left(x_{0}\right)+\phi_{1}\left(x_{0}\right)(h),
$$

is an affine multifunction. We affirm that $A$ is Fréchet differentiable. In effect, given $\varepsilon>0$, there exists $\delta>0$ such that if $\|h\|<\delta$, then

$$
d_{H}\left(\phi_{1}\left(x_{0}+h\right)(z), \phi_{1}\left(x_{0}\right)(z)\right) \leq \tilde{d}\left(\phi_{1}\left(x_{0}+h\right), \phi_{1}\left(x_{0}\right)\right)<\varepsilon,
$$

for all $z \in X$, such that $\|z\| \leq 1$. In particular, for $z=h /\|h\|$, we have

$$
\begin{aligned}
d_{H} & \left(\frac{1}{\|h\|} \phi_{1}\left(x_{0}+h\right)(h), \frac{1}{\|h\|} \phi_{1}\left(x_{0}\right)(h)\right) \\
& =d_{H}\left(\phi_{1}\left(x_{0}+h\right)\left(\frac{h}{\|h\|}\right), \phi_{1}\left(x_{0}\right)\left(\frac{h}{\|h\|}\right)\right)<\varepsilon .
\end{aligned}
$$

This is equivalent to

$$
\begin{aligned}
& \phi_{1}\left(x_{0}+h\right)(h) \subseteq \phi_{1}\left(x_{0}\right)(h)+\varepsilon\|h\| B_{Y}, \\
& \phi_{1}\left(x_{0}\right)(h) \subseteq \phi_{1}\left(x_{0}+h\right)(h)+\varepsilon\|h\| B_{Y} .
\end{aligned}
$$

Now, since $F\left(x_{0}+h\right) \ominus F\left(x_{0}\right)=\phi_{1}\left(x_{0}+h\right)(h)$, we have

$$
\begin{aligned}
F\left(x_{0}+h\right)= & F\left(x_{0}\right)+\phi_{1}\left(x_{0}+h\right)(h) \subseteq F\left(x_{0}\right)+\phi_{1}\left(x_{0}\right)(h) \\
& +\varepsilon\|h\| B_{Y} \subseteq A(h)+\varepsilon\|h\| B_{Y} .
\end{aligned}
$$

Analogously,

$$
\begin{aligned}
A(h)= & F\left(x_{0}\right)+\phi_{1}\left(x_{0}\right)(h) \subseteq F\left(x_{0}\right)+\phi_{1}\left(x_{0}+h\right)(h) \\
& +\varepsilon\|h\| B_{Y} \subseteq F\left(x_{0}+h\right)+\varepsilon\|h\| B_{Y} .
\end{aligned}
$$

The reciprocal of the above theorem is not always true, because the Hukuhara difference does not exist (see Remark 2). But when this difference exists, we have a relationship between Fréchet's differentiability and $\mathrm{CH}$-differentiability.

Theorem 8. If the Hukuhara difference belongs to $b c c Y$ and $F: X \longrightarrow b c c Y$ is a Fréchet differentiable multifunction at $x_{0}$, then $\mathrm{F}$ is $\mathrm{CH}$-differentiable at $x_{0}$.

Proof. Let $A$ be the Fréchet differential of $F$ at $x_{0}$ and $x \in X$. By the Hahn-Banach theorem, there exists a lineal function $\rho_{x}$ $: X \longrightarrow \mathbb{R}$ in $X^{*}$, such that

$$
\rho_{x}(0)=0, \rho_{x}\left(x-x_{0}\right)=\left\|x-x_{0}\right\|_{X}^{2} \text {, and }\left\|\rho_{x}\right\|_{X^{*}}=\left\|x-x_{0}\right\|_{X} .
$$

Then, we can define the function $\phi: X \longrightarrow M A F F_{0}(X$, bcc $Y$ ) by

$$
\phi_{1}(x)= \begin{cases}\frac{1}{\left\|x-x_{0}\right\|_{X}^{2}}\left[\rho_{x}(\cdot)\left(\left(f(x) \Theta_{g} f\left(x_{0}\right)\right) !_{g} A\left(x-x_{0}\right)\right)\right]+A(\cdot), & \text { if } x \neq x_{0}, \\ A(\cdot), & \text { if } x=x_{0} .\end{cases}
$$


This function is continuous at $x_{0}$ (see Example 2). And

$$
\begin{aligned}
\phi_{1}(x)\left(x-x_{0}\right) & =\frac{1}{\left\|x-x_{0}\right\|_{X}^{2}}\left[\rho_{x}\left(x-x_{0}\right)\left(\left(f(x) \Theta_{g} f\left(x_{0}\right)\right) \Theta_{g} A\left(x-x_{0}\right)\right)\right]+A\left(x-x_{0}\right) \\
& =\frac{1}{\left\|x-x_{0}\right\|_{X}^{2}}\left[\left\|x-x_{0}\right\|_{X}^{2}\left(\left(f(x) \Theta_{g} f\left(x_{0}\right)\right) \Theta_{g} A\left(x-x_{0}\right)\right)\right]+A\left(x-x_{0}\right) \\
& =\left(\left(f(x) \Theta_{g} f\left(x_{0}\right)\right) \Theta_{g} A\left(x-x_{0}\right)\right)+A\left(x-x_{0}\right)=f(x) \Theta_{g} f\left(x_{0}\right) .
\end{aligned}
$$

Analogously, for

$$
\phi_{2}(x)= \begin{cases}\frac{1}{\left\|x-x_{0}\right\|_{X}^{2}}\left[\rho_{x}(\cdot)\left(\left(f\left(x_{0}\right) \Theta_{g} f(x)\right) \Theta_{g} A\left(x-x_{0}\right)\right)\right]+A(\cdot), & \text { if } x \neq x_{0}, \\ A(\cdot), & \text { if } x=x_{0} .\end{cases}
$$

we have that $\phi_{2}$ is continuous and $\phi_{2}(x)\left(x-x_{0}\right)=f\left(x_{0}\right) \Theta_{g} f$ $(x)$.

Now, we remember the generalized Hukuhara differentiability.

Definition 5: (see [9]). Let $x_{0} \in \mathbb{R}$ and $F: \mathbb{R} \longrightarrow \mathrm{bcc} Y$ be a multifunction, then the $\mathrm{gH}$-derivative of the multifunction $\mathrm{F}$ at $x_{0}$ is defined as

$$
\partial_{F}\left(x_{0}\right)=\lim _{h \longrightarrow 0} \frac{F\left(x_{0}+h\right) \Theta_{g} F\left(x_{0}\right)}{h} .
$$

If $\partial_{F}\left(x_{0}\right) \in \operatorname{bcc} Y$ satisfying (52) exists, we say that $F$ is generalized Hukuhara differentiable (gH-differentiable for short) at $x_{0}$.

Theorem 9. Let $F: \mathbb{R} \longrightarrow c c Y$ be a multifunction, if $F$ is $C H$ differentiable at $x_{0} \in \mathbb{R}$. Then, $F$ is generalized Hukuhara differentiable at $x_{0} \in \mathbb{R}$.

Proof. Using the CH-differentiability of $F$, there exist multifunctions $\phi_{1}, \phi_{2}: V_{x_{0}} \longrightarrow \operatorname{MAFF}_{0}(\mathbb{R}, \mathrm{bcc} Y)$ continuous at $x$ $\in V_{x_{0}}$ (neighborhood at $\left.x_{0}\right)$, such that $\phi_{1}\left(x_{0}\right)=\phi_{2}\left(x_{0}\right)$ and

$$
\begin{aligned}
& F\left(x_{0}+h\right) \Theta_{g} F\left(x_{0}\right)=\phi_{1}\left(x_{0}+h\right)(h), \\
& F\left(x_{0}\right) \Theta_{g} F\left(x_{0}+h\right)=\phi_{2}\left(x_{0}+h\right)(h),
\end{aligned}
$$

for all $h \in \mathbb{R}$ such that $x_{0}+h \in V_{x_{0}}$. Hence,

$$
\lim _{h \rightarrow 0} \frac{F\left(x_{0}+h\right) \Theta_{g} F\left(x_{0}\right)}{h}=\lim _{h \rightarrow 0} \frac{\phi_{1}\left(x_{0}+h\right)(h)}{h}=\lim _{h \rightarrow 0} \phi_{1}\left(x_{0}+h\right)(1) .
$$

Now, from the continuity of $\phi_{1}$, given $\varepsilon>0$ there exists $\delta>0$ such that, if $|h|<\delta$ then $\tilde{d}\left(\phi\left(x_{0}+h\right), \phi\left(x_{0}\right)\right)<\varepsilon$, in particular

$$
d_{H}\left(\phi\left(x_{0}+h\right)(1), \phi\left(x_{0}\right)(1)\right)<\varepsilon,
$$

thus

$$
\lim _{h \rightarrow 0} \frac{F\left(x_{0}+h\right) \Theta_{g} F\left(x_{0}\right)}{h}=\phi_{1}\left(x_{0}\right)(1) .
$$

Remark 7. Throughout this article, we confine ourselves to multifunctions $F: X \longrightarrow \mathrm{bcc} Y$, as the generalized Hukuhara difference not always exist, to study the inverse of the Theorem (4.5), consider the case $b c c \mathbb{R}=\square$ (where $\square$ denote the set of closed bounded interval of the real line), and the $\mathrm{gH}$ difference exist for any element in $\square$. These multifunctions are called interval-valued functions and are studied in detail in [9].

In the case $\llbracket$, the Hausdorff distance has the following properties:

Proposition 5 (see [9]). For $A, B \in \mathbb{\text { Q }}$, we have

$$
\begin{gathered}
d_{H}(k A, k B)=|k| d_{H}(A, B), \forall k \in \mathbb{R}, \\
d_{H}(A, B)=d_{H}\left(A \Theta_{g} B,\{0\}\right) .
\end{gathered}
$$

Theorem 10. Let $F: \mathbb{R} \longrightarrow \square$ be a multifunction, if $F$ is generalized Hukuhara differentiable at $x_{0} \in \mathbb{R}$, then $F$ is $\mathrm{CH}$ differentiable at $x_{0} \in \mathbb{R}$.

Proof. Using the generalized Hukuhara differentiability of $F$, there is $\partial_{F}\left(x_{0}\right) \in \llbracket$ satisfying (52). We define

$$
\mathscr{A}_{x_{0}}: \mathbb{R} \longrightarrow \mathbb{\square}
$$

given by $\mathscr{A}_{x_{0}}(h)=F\left(x_{0}\right)+h \partial_{F}\left(x_{0}\right)$. By Lemma 4.1, the multifunction (58) is affine, moreover $\mathscr{A}_{x_{0}}(0)=F\left(x_{0}\right)$. On the other hand,

$\lim _{h \rightarrow 0} \frac{d_{H}\left(F\left(x_{0}+h\right), \mathscr{A}_{x_{0}}(h)\right)}{|h|}=\lim _{h \rightarrow 0} \frac{d_{H}\left(F\left(x_{0}+h\right), F\left(x_{0}\right)+h \partial_{F}\left(x_{0}\right)\right)}{|h|}$.

Thus, by Proposition 5, we have

$$
\lim _{h \rightarrow 0} \frac{d_{H}\left(F\left(x_{0}+h\right), \mathscr{A}_{x_{0}}(h)\right)}{|h|}=\lim _{h \rightarrow 0} d_{H}\left(\frac{F\left(x_{0}+h\right) \Theta_{g} F\left(x_{0}\right)}{h}, \partial_{F}\left(x_{0}\right)\right)=0,
$$

since $\lim _{h \longrightarrow 0}\left(F\left(x_{0}+h\right) \Theta_{g} F\left(x_{0}\right) / h\right)=\partial_{F}\left(x_{0}\right)$. Therefore, $F$ is Fréchet differentiable, and by Theorem 8 , is $\mathrm{CH}$ differentiable.

\section{Data Availability}

The datasets generated during and/or analysed during the current study are available from the corresponding author on reasonable request. 


\section{Conflicts of Interest}

The authors declare that there is no conflict of interest regarding the publication.

\section{References}

[1] G. Anichini, "Boundary value problem for multivalued differential equations and controllability," Journal of Mathematical Analysis and Applications, vol. 105, no. 2, pp. 372-382, 1985.

[2] G. Conti, P. Nistri, and P. Zecca, "Controllability problems via set-valued maps," 1991, Recent advances in mathematical theory of systems, control, networks and signa processing, II (Kobe).

[3] J. Ren and J. Wu, "The optimal control problem associated with multi-valued stochastic differential equations with jumps," Nonlinear Analysis: Theory, Methods \& Applications, vol. 86, pp. 30-51, 2013.

[4] A. R. R. Narváez and E. F. Costa, "Average reachability of continuous-time markov jump linear systems and the linear minimum mean square estimator," SIAM Journal on Control and Optimization, vol. 54, no. 4, pp. 2063-2089, 2016.

[5] A. Lasota and A. Strauss, "Asymptotic behavior for differential equations which cannot be locally linearized," Journal of Differential Equations, vol. 10, no. 1, pp. 152-172, 1971.

[6] F. S. De Blasi, "On the differentiability of multifunctions," Pacific Journal of Mathematics, vol. 66, no. 1, pp. 67-81, 1976.

[7] J. Penot, "Differentiability of relations and differential stability of perturbed optimization problems," SIAM Journal on Control and Optimization, vol. 22, no. 4, pp. 529-551, 1984.

[8] M. Michta and J. Motyl, "Differentiable selections of multifunctions and their applications," Nonlinear Analysis: Theory, Methods and Applications, vol. 66, no. 2, pp. 536-545, 2007.

[9] L. Stefanini and B. Bede, "Generalized Hukuhara differentiability of interval-valued functions and interval differential equations," Nonlinear Analysis, vol. 71, no. 3-4, pp. 13111328, 2009.

[10] H. T. Banks and M. Q. Jacobs, "A differential calculus for multifunctions," Journal of Mathematical Analysis and Aplications, vol. 29, no. 2, pp. 246-272, 1970.

[11] V. Gorokhovik and P. Zabreiko, "On Fréchet differentiability of multifunctions," Optimization, (Taylor \& francis), vol. 54, no. 4-5, pp. 391-409, 2005.

[12] B. Bede and S. G. Gal, "Generalizations of the differentiability of fuzzy-number-valued functions with applications to fuzzy differential equations," Fuzzy Sets and Systems, vol. 151, no. 3, pp. 581-599, 2005.

[13] S. E. Amrahov, A. Khastan, N. Gasilov, and A. G. Fatullayev, "Relationship between Bede-Gal differentiable set-valued functions and their associated support functions," Fuzzy Sets and Systems, vol. 295, pp. 57-71, 2016.

[14] S. Kuhn, "The derivative a la Carathéodory," American Mathematical Monthly, vol. 98, no. 1, pp. 40-44, 1991.

[15] G. E. Acosta and G. C. Delgado, "Fréchet vs. Carathéodory," Carathéodory. American Mathematical Monthly, vol. 101, no. 4, pp. 332-338, 1994.

[16] M. Fréchet, La notion de differentielle dnas l'analyse générale, vol. 42 of 3e série, , Annales scientifiques de l'École Normale Supérieure, 1925.
[17] L. Stefanini, "A generalization of Hukuhara difference," in Soft Methods for Handling Variability and Imprecision. Advances in Soft Computing, D. Dubois, M. A. Lubiano, H. Prade, M. Á. Gil, P. Grzegorzewski, and O. Hryniewicz, Eds., vol. 48, pp. 203-210, Springer, Berlin, Heidelberg, 2008.

[18] L. Stefanini, "A generalization of Hukuhara difference and division for interval and fuzzy arithmetic," Fuzzy Sets and Systems, vol. 161, no. 11, pp. 1564-1584, 2010. 